\title{
Betacellulin Regulates Peripheral Nerve \\ Regeneration by Affecting Schwann Cell Migration and Axon Elongation
}

\section{Yaxian Wang}

Nantong University https://orcid.org/0000-0001-5854-9352

Fuchao Zhang

Nantong University

Yunsong Zhang

Nantong University

Qi Shan

Nantong University

Wei Liu

Nantong University

\section{Fengyuan Zhang}

Nantong University

\section{Feiyu Zhang}

Nantong University

Sheng Yi ( $\nabla$ syi@ntu.edu.cn )

Nantong University https://orcid.org/0000-0003-1316-3370

\section{Research article}

Keywords: peripheral nerve injury, betacellulin, Schwann cell migration, axon elongation, nerve regeneration

Posted Date: February 5th, 2021

DOl: https://doi.org/10.21203/rs.3.rs-174606/v1

License: (c) (i) This work is licensed under a Creative Commons Attribution 4.0 International License. Read Full License

Version of Record: A version of this preprint was published at Molecular Medicine on March 25th, 2021. See the published version at https://doi.org/10.1186/s10020-021-00292-5. 


\section{Abstract}

Background Growth factors execute essential biological functions and affect various physiological and pathological processes, including peripheral nerve injury and regeneration. Our previous sequencing analysis found that betacellulin (Btc), an epidermal growth factor protein family member, showed elevated mRNA expressions in the nerve segment after rat peripheral nerve injury, implying the potential involvement of Btc during peripheral nerve repair.

Methods Expression of Btc was examined in Schwann cells. The role of Btc in regulating Schwann cells was investigated by transfecting cultured cells with siRNA segment against Btc or exposed cultured cells with Btc recombinant protein, respectively. The biological functions of Schwann cell-secreted Btc on neurons were also determined. Moreover, the in vivo effect of Btc on Schwann cell migration and axon elongation after rat sciatic nerve injury were further evaluated.

Results Immunostaining images and ELISA readings showed Btc was present in and secreted by Schwann cells. Transwell migration and wound healing observations showed that siRNA against Btc impeded Schwann cell migration while exogenous Btc advanced Schwann cell migration. Besides the regulating effect on Schwann cell phenotype, Btc secreted by Schwann cells might influence neuron behavior and affect axon length. In vivo evidence showed that Btc enhanced axonal regrowth and nerve regeneration after both rat sciatic nerve crush injury and transection injury.

Conclusion Our findings demonstrated Btc-mediated Schwann cell-axon interactions, revealed the essential roles of Btc on Schwann cell migration and axon elongation, and implied the potential application of Btc as a regenerative strategy for treating peripheral nerve injury.

\section{Introduction}

Although peripheral nerves exhibited greater regenerative abilities after nerve injury as compared with central nerves, peripheral nerve injury, especially peripheral nerve injury with long nerve defects, is still a severe health problem [1]. The success of peripheral nerve regeneration relies on the activation of neuronal intrinsic growth capacity and the construction of a permissive microenvironment [2]. Local administration of proteins and molecules with neurotrophic and neuroregenerative properties largely benefits nerve regeneration and is considered as a promising regenerative strategy [3].

Growth factors are natural molecules that function to regulate cellular behaviors. Numerous growth factors, such as nerve growth factor, brain-derived neurotrophic factor, glial cell line-derived neurotrophic factor, and fibroblast growth factors have been identified as essential neurotrophic factors that can be delivered to boost nerve repair $[4,5]$. Our recent study screened critical growth factors that were differentially expressed after rat sciatic nerve injury by the joint use of high-throughput sequencing data and advanced bioinformatic tools and found that besides those well-investigated growth factors, Btc, a gene encoding for betacellulin (Btc), was robustly up-regulated in the injured sciatic nerve segments [6]. 
Btc is a secreted growth factor that belongs to the epidermal growth factor protein family. Btc has been identified as a potent mitogen for different types of cells, including retinal pigment epithelial cells, vascular smooth muscle cells, $\beta$ cells, retinal progenitor cells, and neural stem cells [7-11]. A recent study demonstrated that Btc could also drive the proliferation of Schwann cells, a unique type of glial cells in the peripheral nervous system, implying the critical roles of Btc in the injured nerve segments after peripheral nerve injury [12]. Notably, besides proliferation and myelination, the migration of Schwann cells is also essential for successful peripheral nerve repair as migrating Schwann cells form a cord in the nerve bridge, generate a regenerative microenviroment, and guide axon regeneration $[13,14]$. The functions of Btc on Schwann cell migration, however, have not yet been investigated.

To gain a more comprehensive understanding of the biological roles of growth factor Btc during peripheral nerve regeneration, we cultured primary Schwann cells, transfected cultured Schwann cells with siRNA segment against Btc or exposed cultured Schwann cells with Btc recombinant protein, and performed Transwell migration assay and wound healing assay to observe the effect of Btc on Schwann cell migration. Considering that Btc is a secreted protein, the biological functions of Schwann cellsecreted Btc on neurons were determined. The in vivo effect of Btc on Schwann cell migration and axon elongation after rat sciatic nerve injury were further evaluated.

\section{Materials And Methods \\ Ethical statement}

Sprague-Dawley (SD) rats were obtained from the Experimental Animal Center of Nantong University. Animal experiments were conducted based on Institutional Animal Care Guidelines of Nantong University and approved ethically by the Administration Committee of Experimental Animals, Jiangsu, China.

\section{Schwann cell culture and treatment}

Primary Schwann cells were collected and cultured as previously described [15]. Briefly, cells isolated from neonatal SD rat sciatic nerves were purified with anti-Thy1.1 antibody (Sigma, St. Louis, MO, USA) and rabbit complement (Invitrogen, Carlsbad, CA, USA) and then cultured in DMEM (Gibco, Grand Island, NY, USA) containing 10\% FBS (Gibco), 1\% penicillin and streptomycin (Invitrogen), $2 \mu \mathrm{M}$ forskolin (Sigma), and $10 \mathrm{ng} / \mathrm{ml}$ HRG (R\&D Systems Inc., Minneapolis, MN, USA). For Btc knockdown, cultured primary Schwann cells were transfected with siRNA segments targeting Btc (siRNA sequences: siRNA-1: TCTTCGGAAACATCGCAAA, siRNA-2: CAAGCATTACTGCATCCAT, and siRNA-3:

GAAACCAATGGCTCTCTTT) or a non-targeting negative control (random sequence, RiboBio, Guangzhou, Guangdong, China) for 48 hours using Lipofectamine RNAiMAX transfection reagent (Invitrogen). For Btc protein exposure, Schwann cells were pre-treated with $10 \mathrm{ng} / \mathrm{ml}$ Btc recombinant protein (100-50-20, PeproTech, Rocky Hill, NJ, USA) dissolved in $0.1 \%$ BSA or $0.1 \%$ BSA control for 24 hours. 


\section{Immunofluorescence staining}

Cultured Schwann cells fixed with $4 \%$ paraformaldehyde and exposed to primary antibodies rabbit antiBtc (1:100, PA5-76664, Invitrogen) and mouse anti-S100 (1:100, ab4066, Abcam, Cambridge, MA, USA) followed by reaction with secondary antibodies Alexa Fluor 488-conjugated anti-rabbit (1:400, ab150077, Abcam) and Cy3-conjugated anti-Mouse (1:400, SA00009-1, Proteintech). Cell nuclei were stained with DAPI Fluoromount-G (Southern Biotech, Birmingham, AL, USA). Images were captured using Zeiss Axio Imager M2 (Carl Zeiss Microscopy GmbH, Jena, Germany).

\section{Real-time RT-PCR}

Real-time RT-PCR was performed using RNAs isolated from cultured Schwann cells and SYBR Green Premix Ex Taq (TaKaRa) on a StepOne Real-time PCR System (Applied Biosystems, Foster City, CA, USA). The relative mRNA expression of Btc was measured using the $2^{-\triangle \Delta C t}$ method. Primer sequences were: Btc (forward) 5'-TCTCCAGTGCGTGGTGG-3' and (reverse) 5'-CGAGAGAAGTGGGTTTTCGATT-3' and GAPDH (forward) 5'- ACAGCAACAGGGTGGTGGAC-3' and (reverse) 5'- TTTGAGGGTGCAGCGAACTT-3'.

\section{ELISA}

A total of $6 \times 10^{4}$ Schwann cells transfected with Btc siRNA or siRNA control, respectively, were cultured in DMEM for additionally 24 hours. Schwann cell culture supernatants were collected and passed through a $0.22 \mu \mathrm{m}$ filter (Millipore, Billerica, MA, USA). The amount of secreted Btc protein was determined with a Btc ELISA Kit (ARG81876, Arigo biolaboratories, Hsinchu, Taiwan, China) according to manufacturer's instructions using a Synergy ${ }^{\mathrm{TM}} 2$ Multi-Mode Microplate Reader (BioTek, Burlington, VT, USA).

\section{Transwell migration assay}

Schwann cells suspended in DMEM at a density of $4 \times 10^{4}$ cells $/ \mathrm{ml}$ were transferred to the upper chamber of a Transwell with $8 \mu \mathrm{m}$ pores (Costar, Cambridge, MA, USA). The bottom chamber of the Transwell was filled with cell culture medium with $10 \%$ FBS to drive cell migration. The upper surface of the upper chamber was cleaned after 24 hours and the bottom surface of the upper chamber was stained with $0.1 \%$ crystal violet. Stained crystal violet was dissolved with $33 \%$ acetic acid to measure absorbance using a Synergy ${ }^{\mathrm{TM}} 2$ Multi-Mode Microplate Reader (BioTek). Images were captured using a DMI 3000B inverted microscope (Leica Microsystems, Bensheim, Germany).

\section{Wound healing assay}


Schwann cells suspended in cell culture medium at a density of $2 \times 10^{4}$ cells $/ \mathrm{ml}$ were transferred to a mold chamber with a $1 \mathrm{~mm}$ wide insert. Insert was removed after cell reaching $>95 \%$ confluent to allow cell migration. Remaining cleaned areas were captured using a DMI 3000B inverted microscope (Leica Microsystems) 9 hours later. Three cleaned areas in each image were randomly selected to calculate relative cleaned areas using Image-Pro Plus (Media Cybernetics, Rockville, MD, USA).

\section{Neuron culture}

Briefly, dorsal root ganglia were obtained from neonatal SD rats, cut into pieces, and digested with collagenase I. Isolated cells were re-suspended in 15\% BSA and subjected to centrifuge to collect pellets. Collected primary neurons were cultured in Neurobasal medium (Gibco) containing 2\% B27 supplement (Gibco), 2 mM L-glutamine (ThermoFisher Scientific, Waltham, MA, USA), and 1\% penicillin and streptomycin (Invitrogen) in a humidified $5 \% \mathrm{CO}_{2}$ incubator $37^{\circ} \mathrm{C}$.

Neurons were co-cultured with Schwann cells transfected with Btc siRNA or siRNA control using Transwell co-culture assay as previous described [16]. Briefly, Schwann cells were seeded onto the upper chamber and neurons were seeded onto a PLL-coated glass slide placed on the bottom chamber. After 24-hour co-culture, neurons were fixed with $4 \%$ paraformaldehyde and immunostained with primary antibody mouse anti-neurofilament-200kD (NF-H; 1:200, N2912, Sigma) followed by secondary antibody Cy3-conjugated Goat anti-Mouse IgG (1:400, SA00009-1, Proteintech). Cell nuclei were stained with DAPI Fluoromount-G (Southern Biotech, Birmingham, AL, USA). Images were captured using Zeiss Axio Imager M2 (Carl Zeiss Microscopy $\mathrm{GmbH}$ ). Axon length was determined using Image J (National Institutes of Health, Bethesda, MA, USA).

\section{In vivo experiments}

Adult male SD rats weighting 180-220g were subjected sciatic nerve crush and Btc recombinant protein injection to evaluate the in vivo effects of Btc. After anaesthetization, sciatic nerves of SD rats were exposed and crushed with a forcep. After sciatic nerve crush injury, $100 \mathrm{ng}$ Btc recombinant proteins dissolved in $0.1 \%$ BSA was diluted in $5 \mu \mathrm{l}$ saline. Btc or equal volume of saline was injected into the epineurium at the crush site by using a microsyringe. Rat sciatic nerve segments were collected at 4 days after crush injury, fixed with $4 \%$ paraformaldehyde, cut with a cryostat (Leica Microsystems, Bensheim, Germany) into $12 \mu \mathrm{m}$ thick nerve sections, immunostained with primary antibodies rabbit anti-SCG10 (1:500, NBP1-49461, Novus Biologicals, Littleton, CO, USA) and mouse anti-neurofilament-200kD (NF-H; 1:200, N2912, Sigma) overnight at $4^{\circ} \mathrm{C}$, and exposed to secondary antibodies Alexa Fluor 488-conjugated anti-mouse secondary antibody (1:400, ab150113, Abcam, Cambridge, MA, USA) and Alexa Fluor 555conjugated anti-rabbit secondary antibody (1:400, ab150078, Abcam) for 2 hours at room temperature. Images were captured using Zeiss Axio Imager M2 (Carl Zeiss Microscopy GmbH). 
Another group of SD rat were subjected sciatic nerve transection to generate a $6 \mathrm{~mm}$ nerve gap. A silicone tube filled with $100 \mathrm{ng}$ Btc recombinant protein dissolved in $0.1 \%$ BSA and $8 \mu$ saline or equal volume of saline was implanted to bridge the nerve gap. Rat sciatic nerve segments collected at 10 days after transection were immunostained with primary antibodies mouse anti-NF-H and rabbit anti-S100 1 (1:200, S2644, Sigma) and secondary antibodies Alexa Fluor 488-conjugated anti-mouse secondary antibody and Alexa Fluor 555-conjugated anti-rabbit secondary antibody. Cell nucleus was stained with Hoechst 33342 (ab228551, Abcam). Images were captured using Zeiss Axio Imager M2 (Carl Zeiss Microscopy $\mathrm{GmbH})$. Axon elongation length and Schwann cell migration distance were calculated using Zeiss Microscopy Software ZEN 2.3.

\section{Statistic analysis}

Summarized outcomes were demonstrated as means \pm SEM from 3-4 paired experiments. Student's t-test or multiple t-tests was used for comparisons. A p-value $<0.05$ was considered as significantly different.

\section{Results}

\section{Btc stimulated Schwann cell migration}

Isolated and cultured Schwann cells were immunostained with Schwann cell marker S100 $\beta$ to assess cell purity. Immunofluorescence images showed that the ratio of the number of $S 100 \beta$-positive cells to that of DAPI-positive cells was $>95 \%$, indicating that we achieved Schwann cells with high purity. Further immunostaining of cultured Schwann cells with Btc showed that the majority of Btc-positive signals overlapped with $\mathrm{S} 100 \beta$-positive signals, suggesting that Btc protein was expressed and present in Schwann cells (Figure 1).

Schwann cells were then transfected with siRNA against Btc to evaluate the functional effects of reduced Btc expression. Transfection of Schwann cells with three siRNA against Btc (designated as siRNA-1, siRNA-2, and siRNA-3) all successfully decreased the mRNA abundance of Btc (Figure 2A). Segment siRNA-3 achieved a relatively high and stable silencing efficiency and was subsequently used for Btc knockdown. Following transfection, Schwann cells were seeded onto the upper chamber of a Transwell to observe cellular migration ability. Compared with cell transfected with si-RNA control, less Schwann cells were observed to be migrated towards the bottom chamber (Figure 2B). The migration ability of Schwann cells was further examined by generating an equal wide blank space and measuring the area of left blank space at 9 hours after cell culture. Remaining cleaned area was robustly largely in Schwann cells transfected with siRNA against Btc as compared with Schwann cells transfecting with si-RNA control, demonstrating that (Figure 2C).

Besides cell transfection, Schwann cells were also directly exposed to Btc recombinant protein. On the contrary to cells transfected with siRNA against Btc, following Btc recombinant protein treatment, the amount of migrated Schwann cells was increased (Figure 3A) while left cleaned area after wound healing 
was decreased (Figure 3B). These observations showed that Btc would promote the migration of Schwann cells.

\section{Schwann cell-secreted Btc stimulated axon elongation}

ELISA was performed to determine the amount of Btc in the supernatant of cultured Schwann cells. ELISA readings revealed that certain level of Btc protein can be detected while transfection of Schwann cells with siRNA against Btc largely reduced the secretion of Btc protein (Figure 4A).

Schwann cells were then in-directly co-cultured with neurons to examine the effect of secreted Btc protein on neuron behaviors (Figure 4B). Neurons co-cultured with Schwann cells transfected with siRNA control possessed much longer axons as compared with neurons co-cultured with Schwann cells transfected with siRNA against Btc, implying that Schwann cell-secreted Btc could promote the elongation of axons (Figure 4C).

\section{Btc promoted the repair of injured peripheral nerves}

In addition to these in vitro examinations, Btc recombinant protein was directly applied to SD rats to observe the in vivo effect of Btc after peripheral nerve injury. First, rat sciatic nerves subjected crush injury were injected with Btc recombinant protein or saline control. Injured sciatic nerve segments were collected at 4 days after nerve crush injury and immunostained with axon markers SCG10 and NF-H. In both Btc recombinant protein-treated group and saline-treated group, regenerated axons grow across the crushed site towards their target organs. However, the intensity of SCG10 seemed to be higher at multiple measured positions in Btc recombinant protein-treated group, especially 1,2, and $4 \mathrm{~mm}$ from the crush site (Figure 5).

Besides sciatic nerve crush, another group of animals were subjected to a $6 \mathrm{~mm}$ nerve transection, a more severe injury. A silicone tube injected with Btc recombinant protein or saline control was used to bridge rat sciatic nerve gap. A certain level of Schwann cell migration and axon elongation were observed in both groups at 10 days after surgery (Figure 6A \& 6B). But rats treated with silicone tubes injected with Btc recombinant protein seemed to have larger amount of Schwann cells and relatively well-formed Schwann cell-cord in the nerve bridge (Figure 6B and 6b) compared to rats receiving saline treatment (Figure 6A and $6 a$ ). Summarized data also showed that the length of regeneration nerve fibers and the migrating distance of Schwann cells were longer in Btc-treated rats (Figure 6C and 6D). These observations indicated that Btc protein would encourage axon elongation and nerve regeneration after peripheral nerve crush injury.

\section{Discussion}


In consideration of the increasing cases of peripheral nerve injury and the inherent disadvantage of autologous nerve grafting, tissue engineered nerve grafts have been more widely applied to treat peripheral nerve injury [5]. Multiple strategies, such as the modulation of the components/topographic structures of neural scaffolds and the incorporation of stem cells have been made to optimize tissue engineered nerve grafts [17-19]. Emerging studies demonstrated that growth factors contain strong postinjury effects for triggering peripheral nerve regeneration [20,21]. Therefore, identifying novel growth factors that promote nerve regeneration will further contribute to the construction of growth factorcombined tissue engineered nerve grafts and be beneficial to the treatment of peripheral nerve injury.

A series of growth factors were discovered to be dysregulated after peripheral nerve injury [6]. The mRNA expressions of $B t c$, among these dysregulated growth factors, was kept up-regulated in the injured nerve stumps at all tested time points (1, 4, 7, and 14 days after nerve injury), implying the potential effect of Btc on Schwann cell phenotype modulation and peripheral nerve regeneration.

Therefore, in the current study, we investigated the biological functions of Btc on peripheral nerve regeneration by using cultured Schwann cells and sciatic nerve injury models. Observations from both Transwell migration assay and wound healing assay showed that siRNA against Btc and Btc recombinant protein decreased and increased the migration rate of Schwann cells, respectively. Consistent with in vitro observations, accelerated Schwann cell migration was also detected after the in vivo application of Btc recombinant protein. These observations, together with a previous finding which demonstrated that Btc stimulated the proliferation of myelinating Schwann cells and induced myelin formation [12], revealed the comprehensive effect of Btc on essential regenerative phenotypes of Schwann cells, i.e., proliferation, migration, and myelination.

Schwann cells not only generate a permissive microenviroment for peripheral nerve regeneration and guide regenerating axonal sprout via forming regeneration tracks, but also provide indispensable supports for neuron survival and growth core formation via producing and secreting trophic factors [2225]. For example, it has been demonstrated that genetic modulated-Schwann cells that up-regulates trophic factors not only facilitated Schwann cell migration but also stimulated neurite outgrowth [26]. Our current study revealed that Schwann cells could secret Btc to their supernatants and Schwann cellsecreted Btc could influence neuron behaviors and promote neurite outgrowth. In vivo observations of the application of Btc at the injury site further demonstrated the promoting role of Btc on axon growth and elongation after nerve injury using both a mild crush injury model and a more severe transection injury model. Notably, previously, our lab mates immobilized nerve growth factor with biomaterials and found that nerve growth factor-incorporated bio-scaffolds could advance nerve regeneration through affecting both Schwann cells and neurons $[27,28]$. Therefore, Btc protein or Btc-over expressing Schwann cells may be combined with neural scaffolds to generate Btc-based tissue engineering nerve grafts for the treatment of peripheral nerve regeneration.

\section{Conclusions}


In summary, our current study illuminated that Btc, an elevated growth factors after peripheral nerve injury, could enhance Schwann cell migration, axon elongation, and nerve regeneration. Our study revealed Btc as a regenerative neurotrophic factor, expanded the understanding of the involvement of growth factors during peripheral nerve regeneration, and proposed the potential usage of Btc in the treatment of severe peripheral nerve injury.

\section{Declarations}

\section{Acknowledgements}

Not applicable.

\section{Authors' contributions}

Conceived and designed the experiments: YW SY. Experiment conductance and data analyses: YW FZ YZ QS WL FZ FZ SY. Contributed reagents/materials/analysis tools: SY. Wrote the manuscript: YW SY.

\section{Availability of supporting data}

Not applicable.

\section{Ethical Approval and Consent to participate}

Animal experiments were conducted based on Institutional Animal Care Guidelines of Nantong University and approved ethically by the Administration Committee of Experimental Animals, Jiangsu, China.

\section{Funding}

This study was supported by the National Natural Science Foundation of China [81901257], the Natural Science Foundation of Jiangsu Province [BK20180951 and BK20202013], the National College Student Innovation Training Program, the Natural Science Research Project Funded by Nantong University [20ZH376, 18B26 and 17ZY12], and Priority Academic Program Development of Jiangsu Higher Education Institutions [PAPD].

\section{Competing interests}

The authors declare that they have no conflict of interest. 


\section{Consent for publication}

Not applicable.

\section{References}

1. Aguilar ZP. (2012) Nanomaterials for Medical Applications.

2. Chen ZL, Yu WM, Strickland S. Peripheral regeneration. Annu Rev Neurosci. 2007;30:209-33. doi:10.1146/annurev.neuro.30.051606.094337.

3. Tajdaran K, Chan K, Gordon T, Borschel GH. Matrices, scaffolds, and carriers for protein and molecule delivery in peripheral nerve regeneration. Exp Neurol. 2019;319:112817. doi:10.1016/j.expneurol.2018.08.014.

4. Gu X, Ding F, Yang Y, Liu J. Construction of tissue engineered nerve grafts and their application in peripheral nerve regeneration. Prog Neurobiol. 2011;93(2):204-30.

doi:10.1016/j.pneurobio.2010.11.002.

5. Gu X, Ding F, Williams DF. Neural tissue engineering options for peripheral nerve regeneration. Biomaterials. 2014;35(24):6143-56. doi:10.1016/j.biomaterials.2014.04.064.

6. Zhang R, Zhang Y, Yi S. (2019) Identification of critical growth factors for peripheral nerve regeneration. RSC Advances 9.

7. Huotari MA, Palgi J, Otonkoski T. Growth factor-mediated proliferation and differentiation of insulinproducing INS-1 and RINm5F cells: identification of betacellulin as a novel beta-cell mitogen. Endocrinology. 1998;139(4):1494-9. doi:10.1210/endo.139.4.5882.

8. Shing Y, Christofori G, Hanahan D, Ono Y, Sasada R, Igarashi K, Folkman J. Betacellulin: a mitogen from pancreatic beta cell tumors. Science. 1993;259(5101):1604-7. doi:10.1126/science.8456283.

9. Zhang D, Shen B, Zhang Y, Ni N, Wang Y, Fan X, Sun H, Gu P. Betacellulin regulates the proliferation and differentiation of retinal progenitor cells in vitro. J Cell Mol Med. 2018;22(1):330-45. doi: $10.1111 / \mathrm{jcmm} .13321$.

10. Oh YS, Shin S, Lee YJ, Kim EH, Jun HS. Betacellulin-induced beta cell proliferation and regeneration is mediated by activation of ErbB-1 and ErbB-2 receptors. PLoS One. 2011;6(8):e23894. doi:10.1371/journal.pone.0023894.

11. Gomez-Gaviro MV, Scott CE, Sesay AK, Matheu A, Booth S, Galichet C, Lovell-Badge R. Betacellulin promotes cell proliferation in the neural stem cell niche and stimulates neurogenesis. Proc Natl Acad Sci U S A. 2012;109(4):1317-22. doi:10.1073/pnas.1016199109.

12. Vallieres N, Barrette B, Wang LX, Belanger E, Thiry L, Schneider MR, Filali M, Cote D, Bretzner F, Lacroix S. Betacellulin regulates schwann cell proliferation and myelin formation in the injured mouse peripheral nerve. Glia. 2017;65(4):657-69. doi:10.1002/glia.23119.

13. Chen B, Chen Q, Parkinson DB, Dun XP. Analysis of Schwann Cell Migration and Axon Regeneration Following Nerve Injury in the Sciatic Nerve Bridge. Front Mol Neurosci. 2019;12:308. 
doi:10.3389/fnmol.2019.00308.

14. Clements MP, Byrne E, Camarillo Guerrero LF, Cattin AL, Zakka L, Ashraf A, Burden JJ, Khadayate S, Lloyd AC, Marguerat S, Parrinello S. The Wound Microenvironment Reprograms Schwann Cells to Invasive Mesenchymal-like Cells to Drive Peripheral Nerve Regeneration. Neuron. 2017;96(1):98-114 e117. doi:10.1016/j.neuron.2017.09.008.

15. Yi S, Liu Q, Wang X, Qian T, Wang H, Zha G, Yu J, Wang P, Gu X, Chu D, Li S. (2019) Tau modulates Schwann cell proliferation, migration and differentiation following peripheral nerve injury. J Cell Sci 132 (6). doi:10.1242/jcs.222059.

16. Zhang Z, Yu B, Gu Y, Zhou S, Qian T, Wang Y, Ding G, Ding F, Gu X. Fibroblast-derived tenascin-C promotes Schwann cell migration through beta1-integrin dependent pathway during peripheral nerve regeneration. Glia. 2016;64(3):374-85. doi:10.1002/glia.22934.

17. Yi S, Ding F, Gong L, Gu X. Extracellular Matrix Scaffolds for Tissue Engineering and Regenerative Medicine. Curr Stem Cell Res Ther. 2017;12(3):233-46.

doi:10.2174/1574888X11666160905092513.

18. Yi S, Xu L, Gu X. Scaffolds for peripheral nerve repair and reconstruction. Exp Neurol. 2019;319:112761. doi:10.1016/j.expneurol.2018.05.016.

19. Yi S, Zhang Y, Gu X, Huang L, Zhang K, Qian T, Gu X. Application of stem cells in peripheral nerve regeneration. Burns Trauma. 2020;8:tkaa002. doi:10.1093/burnst/tkaa002.

20. Onger ME, Delibas B, Turkmen AP, Erener E, Altunkaynak BZ, Kaplan S. The role of growth factors in nerve regeneration. Drug Discov Ther. 2017;10(6):285-91. doi:10.5582/ddt.2016.01058.

21. Li R, Li DH, Zhang HY, Wang J, Li XK, Xiao J. Growth factors-based therapeutic strategies and their underlying signaling mechanisms for peripheral nerve regeneration. Acta Pharmacol Sin. 2020;41(10):1289-300. doi:10.1038/s41401-019-0338-1.

22. Jessen KR, Mirsky R. The repair Schwann cell and its function in regenerating nerves. J Physiol. 2016;594(13):3521-31. doi:10.1113/JP270874.

23. Mahar M, Cavalli V. Intrinsic mechanisms of neuronal axon regeneration. Nat Rev Neurosci. 2018;19(6):323-37. doi:10.1038/s41583-018-0001-8.

24. Allodi I, Udina E, Navarro X. Specificity of peripheral nerve regeneration: interactions at the axon level. Prog Neurobiol. 2012;98(1):16-37. doi:10.1016/j.pneurobio.2012.05.005.

25. Min Q, Parkinson DB, Dun XP. Migrating Schwann cells direct axon regeneration within the peripheral nerve bridge. Glia. 2021;69(2):235-54. doi:10.1002/glia.23892.

26. Huang L, Quan X, Liu Z, Ma T, Wu Y, Ge J, Zhu S, Yang Y, Liu L, Sun Z, Huang J, Luo Z. c-Jun genemodified Schwann cells: upregulating multiple neurotrophic factors and promoting neurite outgrowth. Tissue Eng Part A. 2015;21(7-8):1409-21. doi:10.1089/ten.TEA.2014.0416.

27. Li G, Xiao Q, Zhang L, Zhao Y, Yang Y. Nerve growth factor loaded heparin/chitosan scaffolds for accelerating peripheral nerve regeneration. Carbohydr Polym. 2017;171:39-49.

doi:10.1016/j.carbpol.2017.05.006. 
28. Yang Y, Zhao W, He J, Zhao Y, Ding F, Gu X. Nerve conduits based on immobilization of nerve growth factor onto modified chitosan by using genipin as a crosslinking agent. Eur J Pharm Biopharm. 2011;79(3):519-25. doi:10.1016/j.ejpb.2011.06.008.

\section{Figures}
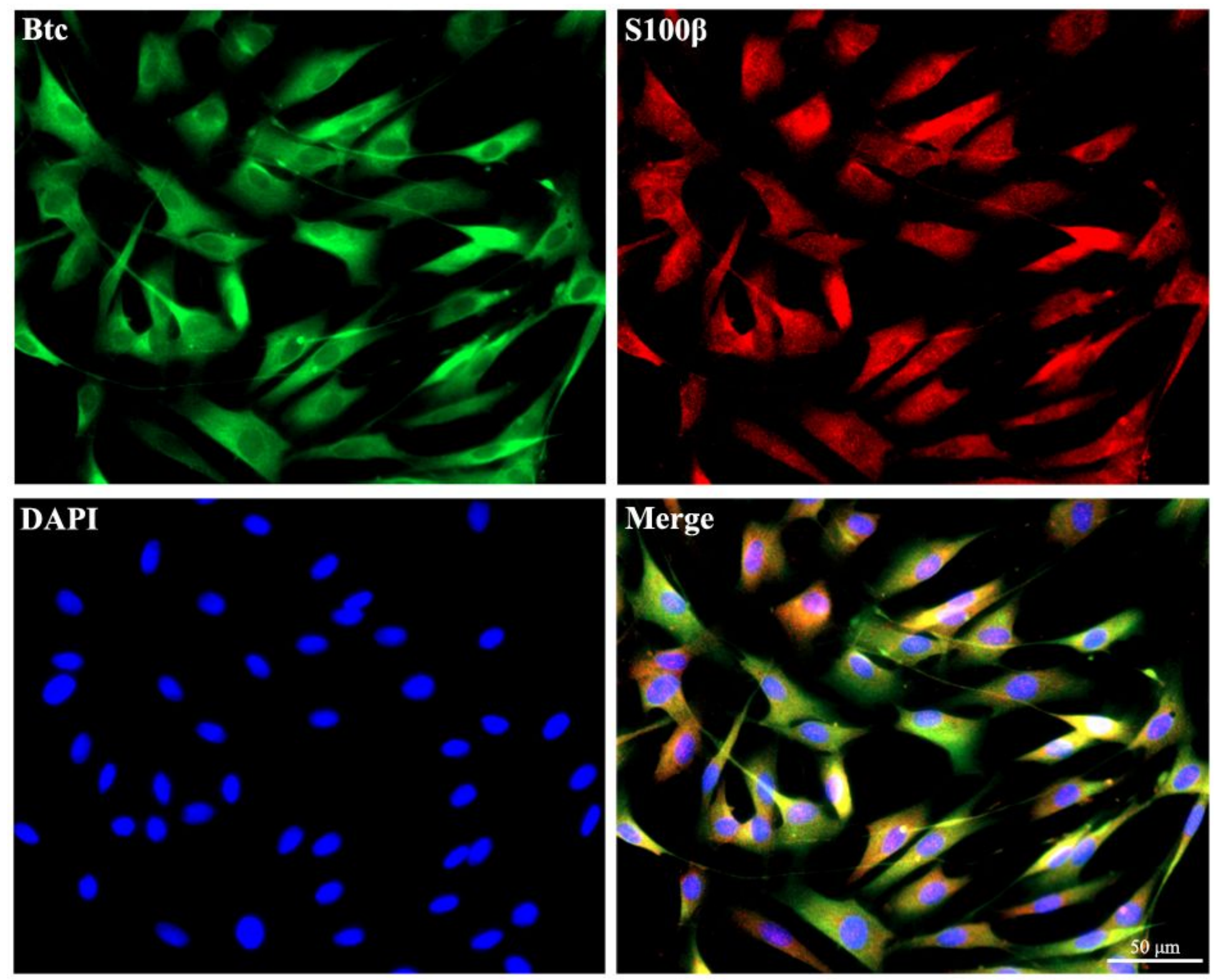

\section{Figure 1}

Immunostaining of Btc in cultured Schwann cells. Red color indicated $\mathrm{S} 100 \beta$, green color indicated Btc, and blue color indicated nucleus. Scale bars indicated $50 \mu \mathrm{m}$. 
A
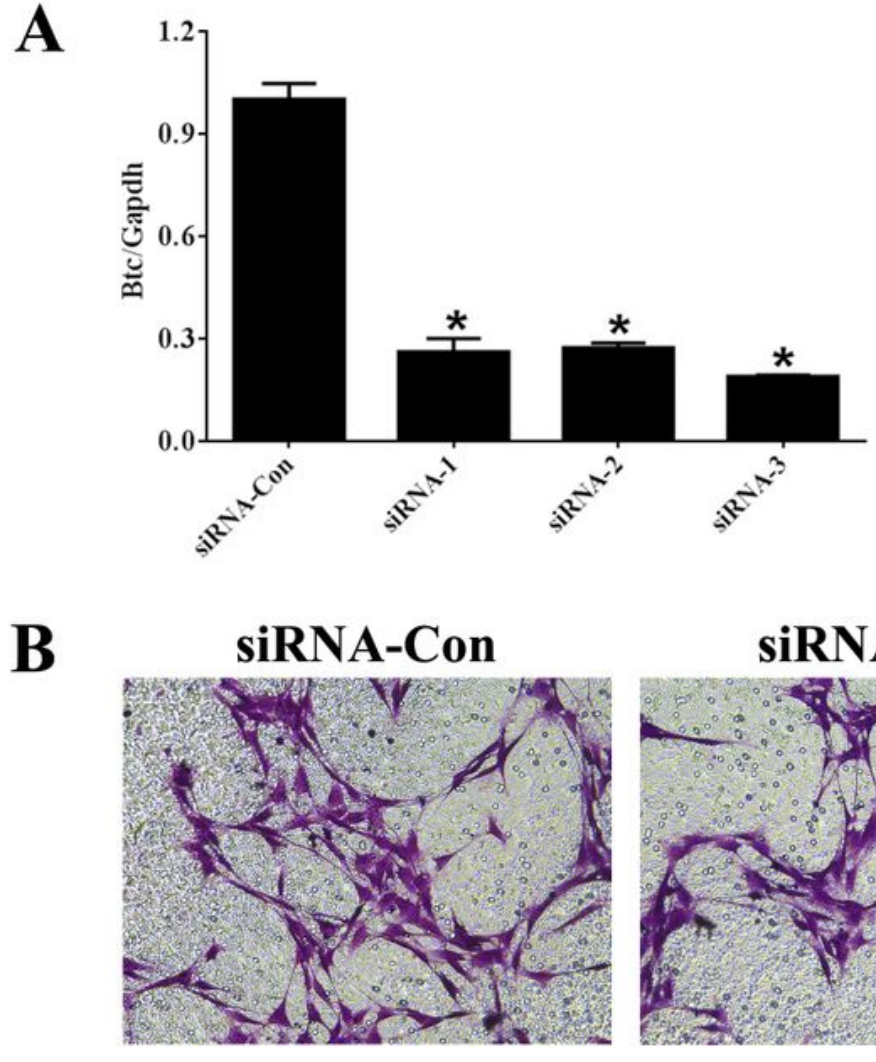

C
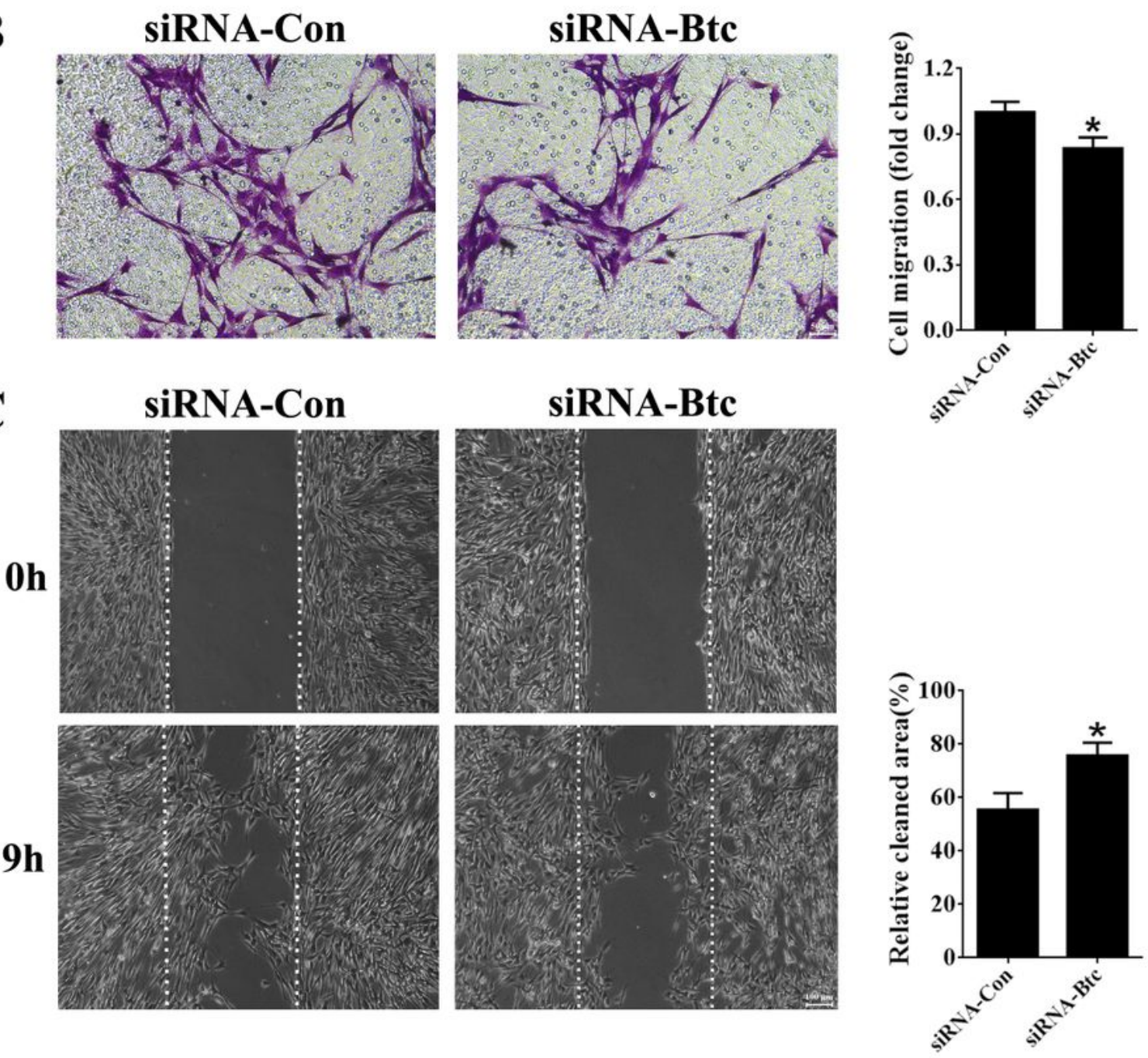

Figure 2

The in vitro effect of siRNA against Btc on Schwann cell migration. (A) The transfection efficiency of siRNA-Btc. $n=3 .{ }^{*}, p$-value $<0.05$ versus siRNA control. (B) Representative Transwell migration images and normalized averaged migration rate of Schwann cells transfected with siRNA control and siRNA-Btc. Scale bars represented $50 \mu \mathrm{m} . \mathrm{n}=3 .{ }^{*}$, $\mathrm{p}$-value $<0.05$ versus siRNA control. (C) Representative wound 

and siRNA-Btc. Scale bars represented $100 \mu \mathrm{m} . \mathrm{n}=4$. *, $\mathrm{p}$-value $<0.05$ versus siRNA control.

A

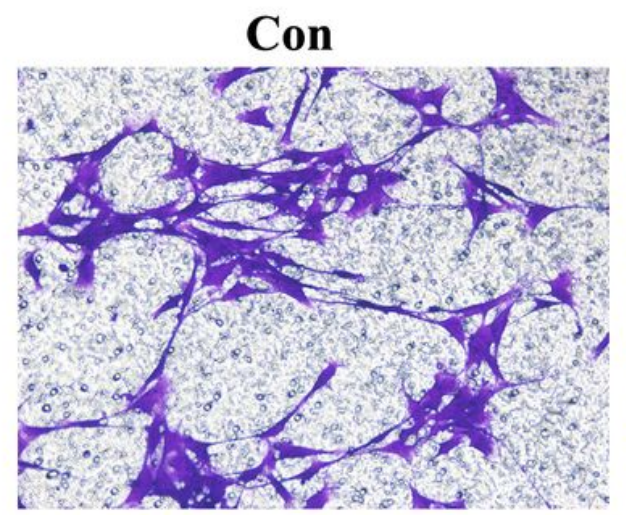

B
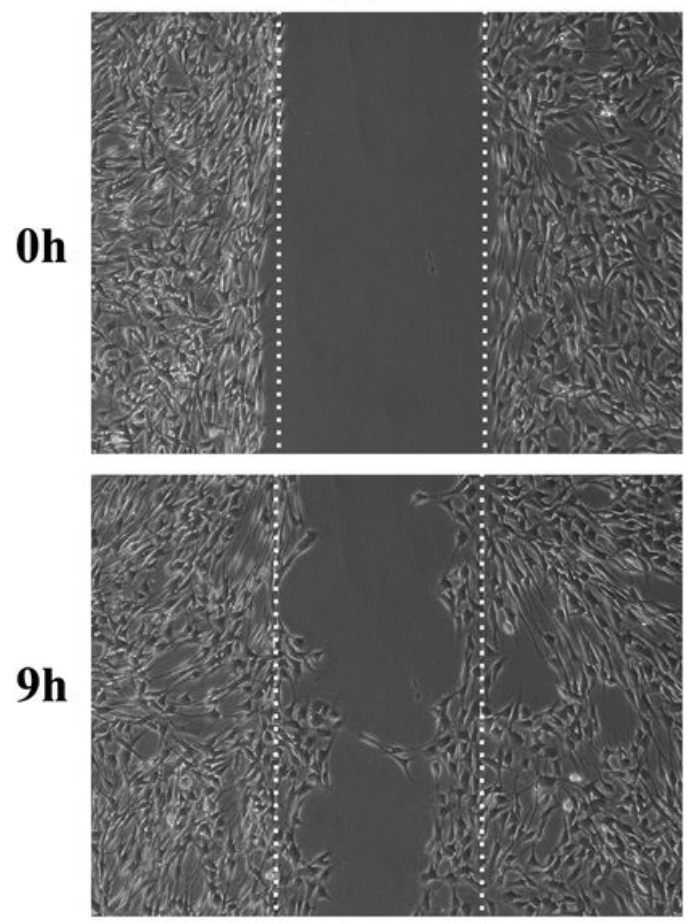

Btc

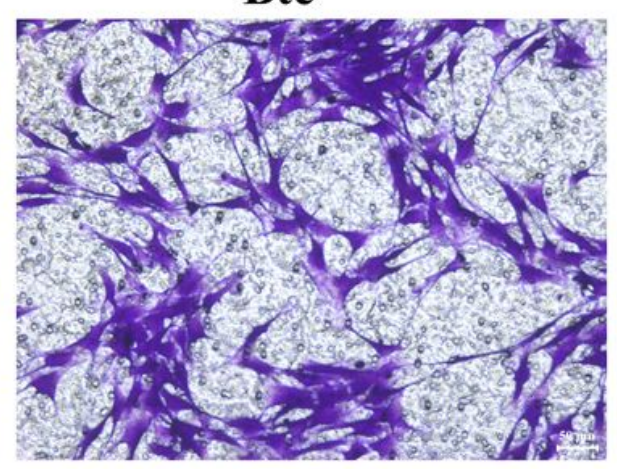

Btc
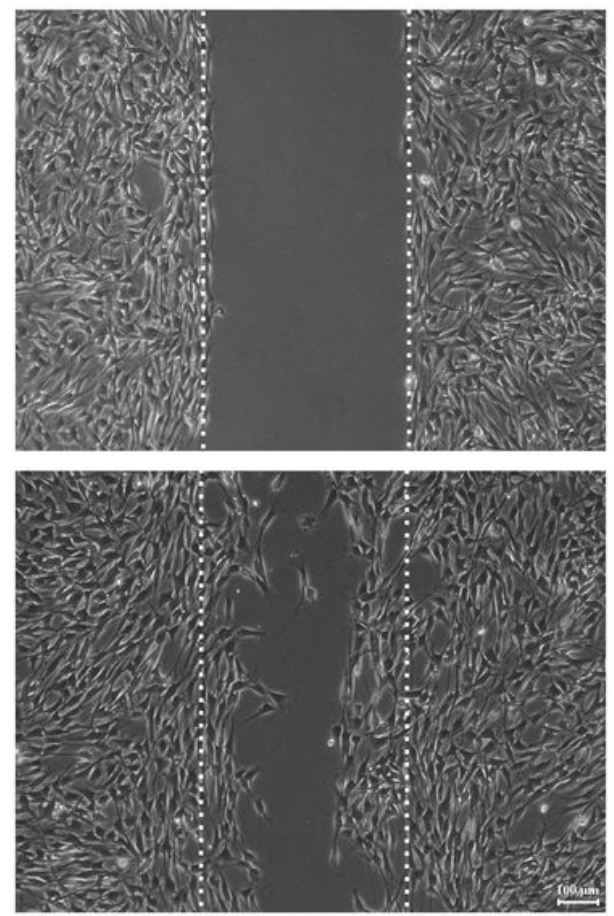
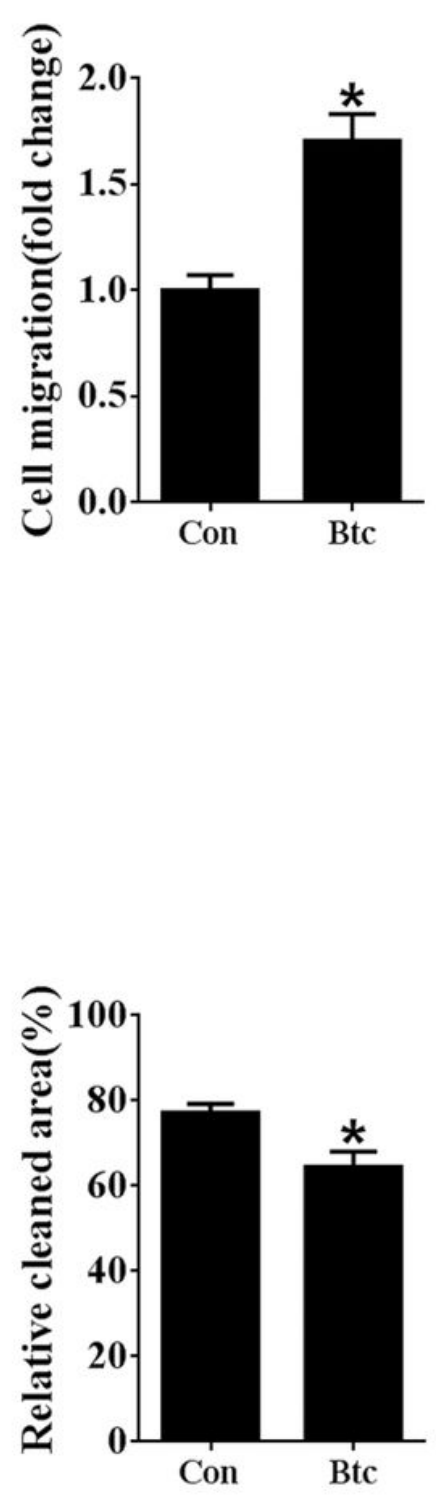

\section{Figure 3}

The in vitro effect of Btc recombinant protein on Schwann cell migration. (A) Representative Transwell migration images and normalized averaged migration rate of Schwann cells treated with or without Btc recombinant protein. Scale bars represented $50 \mu \mathrm{m} . n=3 .{ }^{*}, \mathrm{p}$-value $<0.05$ versus Btc-non-treated cells. (B) Representative wound healing images and normalized averaged cleaned area of Schwann cells treated with or without Btc recombinant protein. Scale bars represented $100 \mu \mathrm{m} . \mathrm{n}=3$. *, $\mathrm{p}$-value $<0.05$ versus Btcnon-treated cells 
A

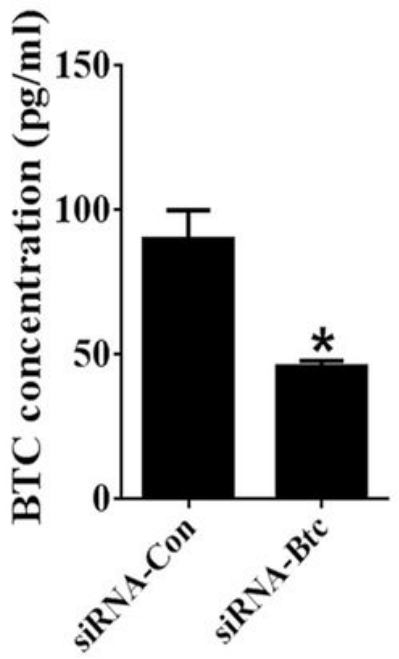

C

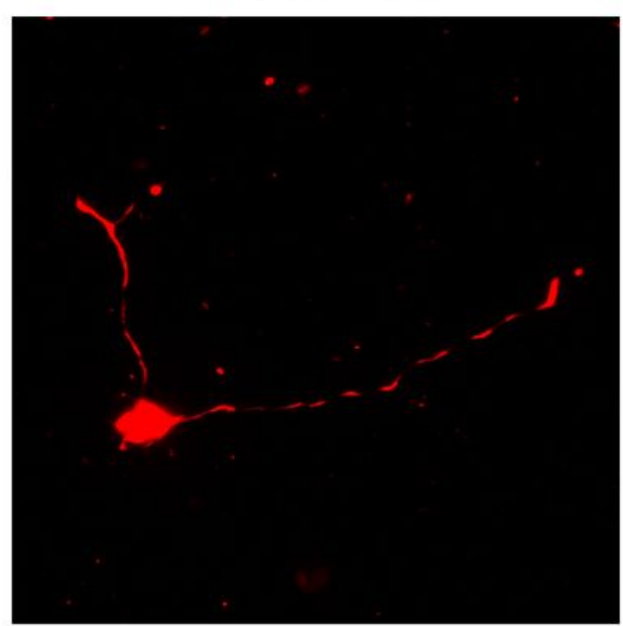

B

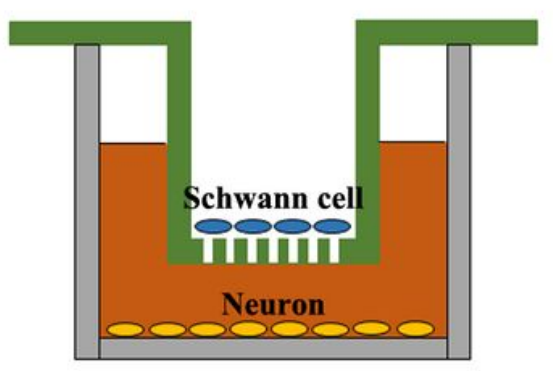

SiRNA-Btc

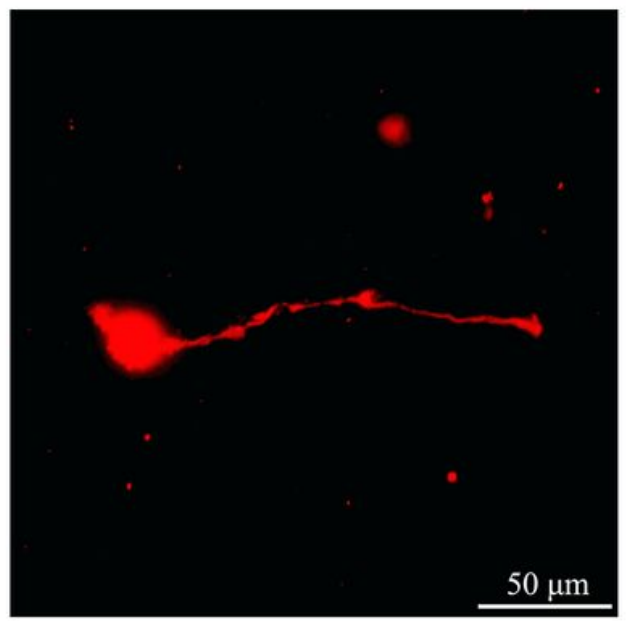

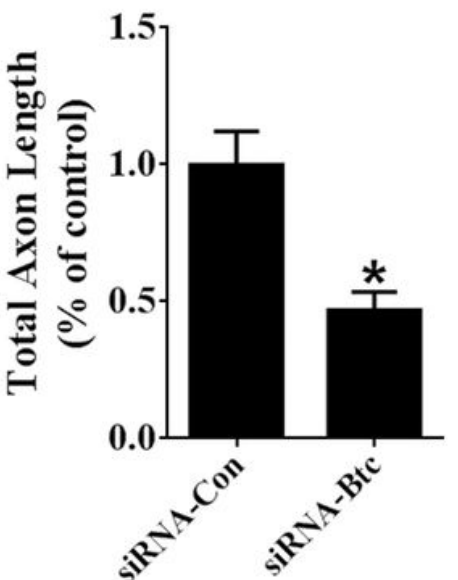

\section{Figure 4}

The in vitro effect of Schwann cell-secreted Btc on axon elongation. (A) The amount of Schwann cellsecreted Btc in Schwann cells transfected with siRNA control and siRNA-Btc. $n=3$. *, $p$-value $<0.05$ versus siRNA control. (B) The schematic diagram of the co-culture of Schwann cells and neurons. (C) Representative immunofluorescence images and normalized averaged axon length of neurons cocultured with transfected with siRNA control and siRNA-Btc. Scale bars represented $50 \mu \mathrm{m} . \mathrm{n}=3$. *, p-value $<0.05$ versus neurons co-cultured with Schwann cells transfected with siRNA control. 

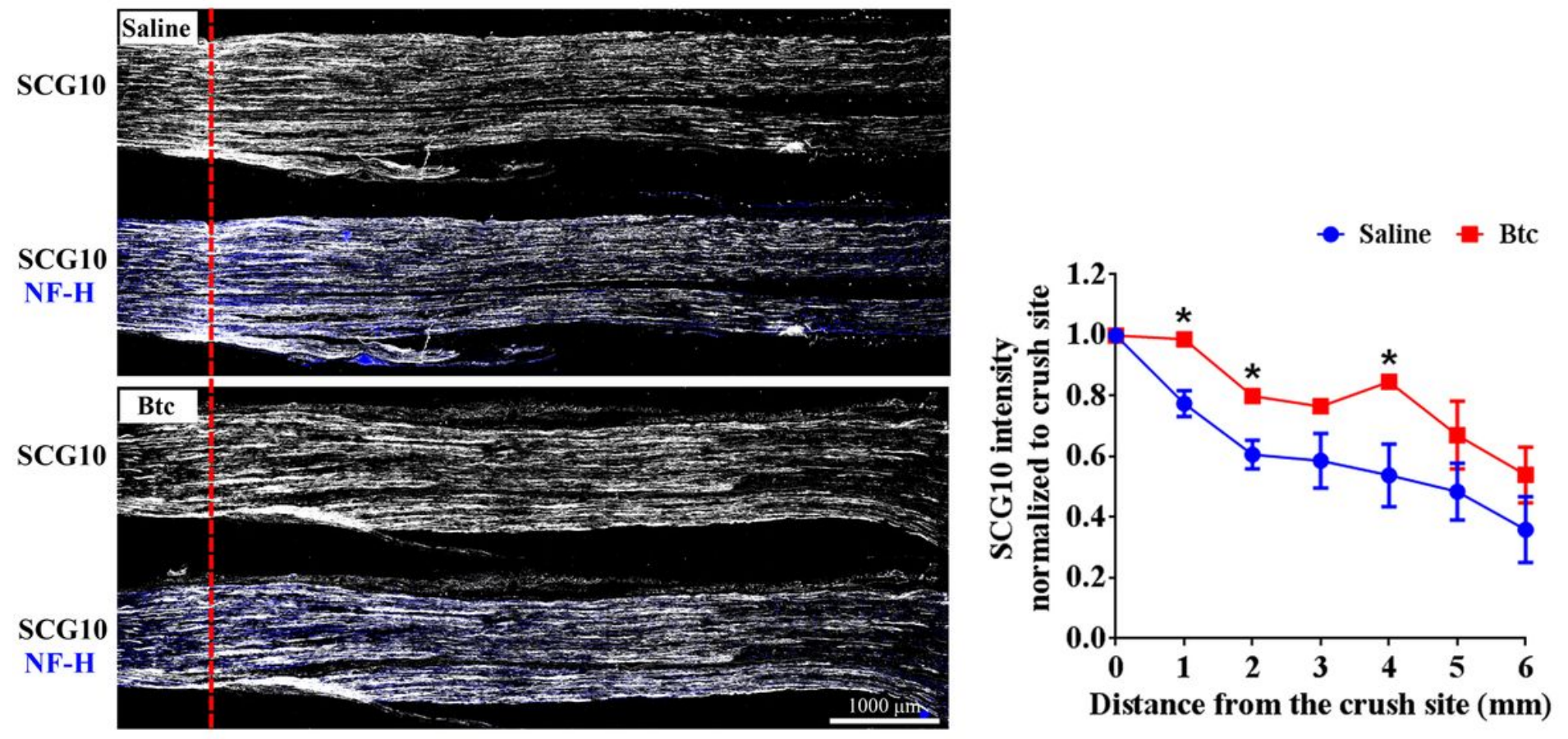

\section{Figure 5}

The in vivo effect of Btc recombinant protein after rat sciatic nerve crush. Representative immunofluorescence images of rat sciatic nerve segments treated with saline control or Btc recombinant protein at 4 days after nerve crush. Averaged intensity of SCG10 at 1, 2, 3, 4, 5, and $6 \mathrm{~mm}$ from the crush site were normalized to its intensity at the crush site. Scale bar indicated $1000 \mu \mathrm{m} . n=3 .{ }^{*}, p$-value $<0.05$ versus saline control. 

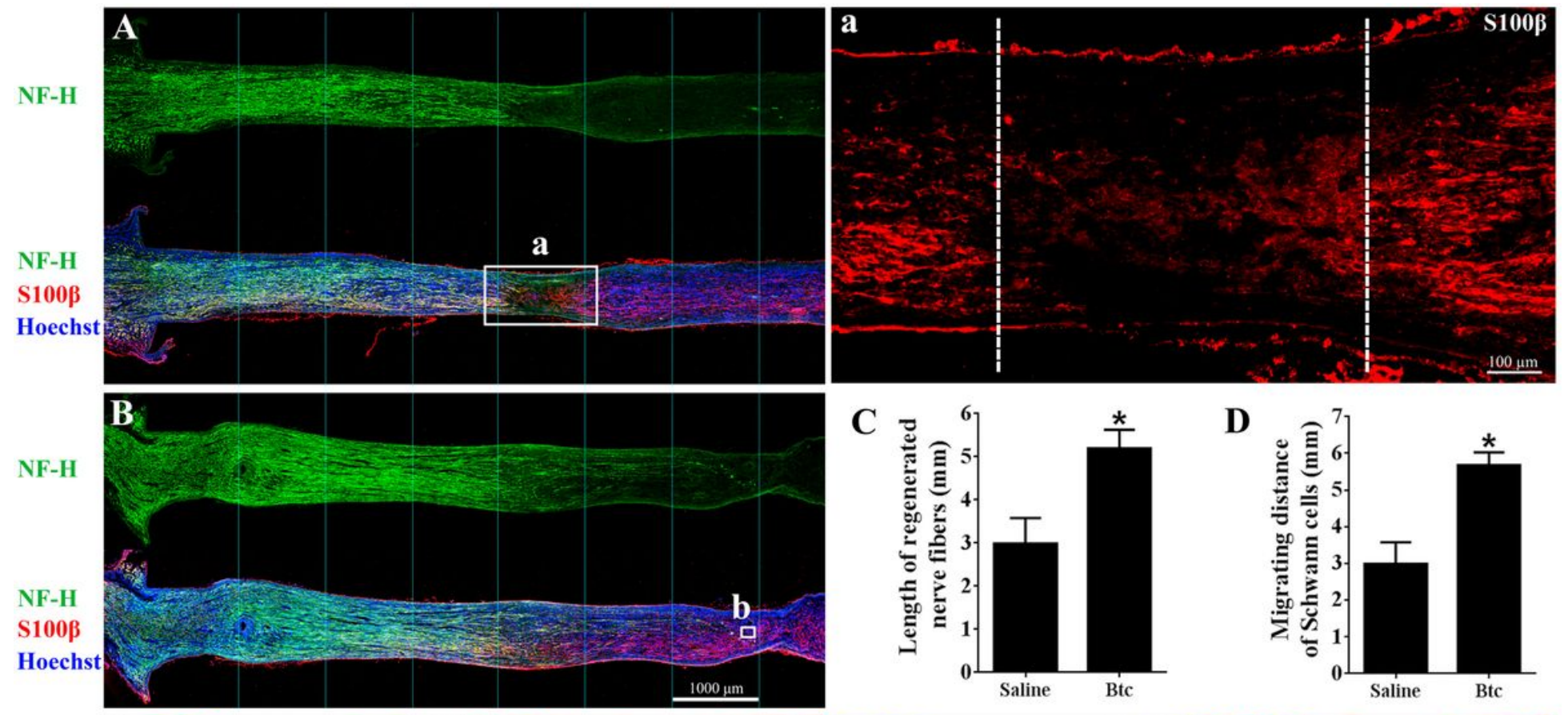

\section{C}

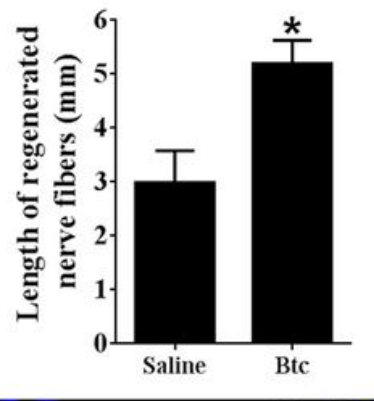

D

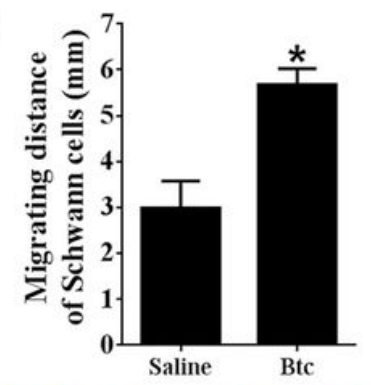

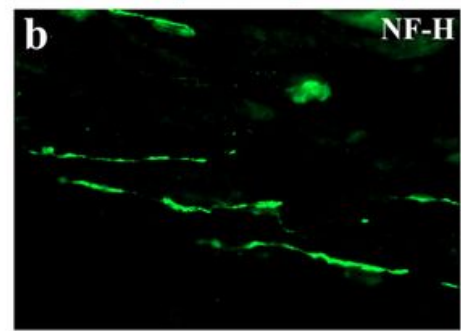
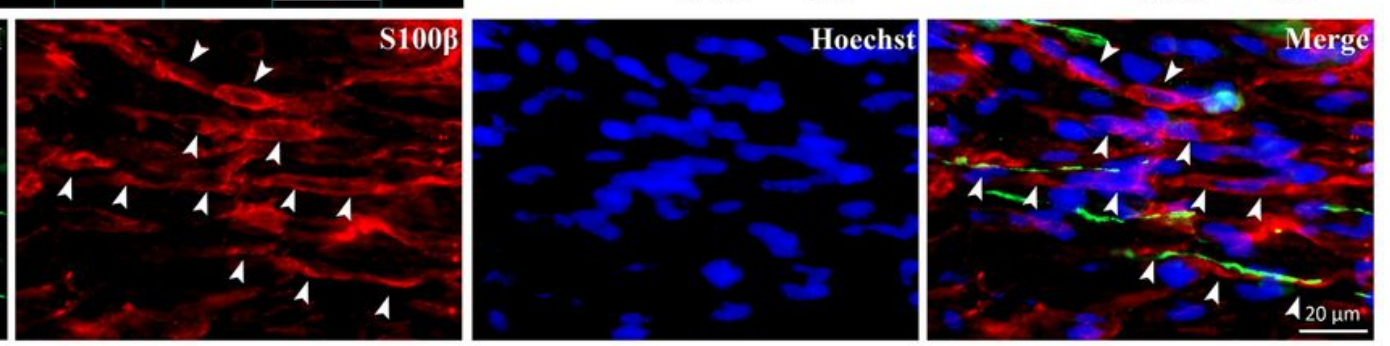

Figure 6

The in vivo effect of Btc recombinant protein after rat sciatic nerve transection and silicone bridging. Representative immunofluorescence images of rat sciatic nerve segments treated with $(A)$ saline control or (B) Btc recombinant protein at 10 days after nerve transection and silicone bridging. Green color indicated NF-H, red color indicated $\mathrm{S} 100 \beta$, and blue color indicated nucleus. Boxed areas in (A and B) were shown in higher magnifications in ( $a$ and $b$ ), respectively. Arrowheads indicated Schwann cellformed cord in the nerve bridge. Scale bars indicated $1000 \mu \mathrm{m}$ in (A and B) and indicated $100 \mu \mathrm{m}$ in (a) and $20 \mu \mathrm{m}$ in (b). (C) Averaged length of regeneration nerve fibers. $n=3 . *$, $p$-value $<0.05$ versus saline control. (D) Averaged migrating distance of Schwann cells. $n=3$. *, $p$-value $<0.05$ versus saline control. 